\title{
Learning the C-A-Bs: Resuscitation Training at Rwanda Military Hospital
}

\author{
Kathryn Norgang ${ }^{1}$, Auni Idi Muhire ${ }^{1}$, Sarah Howrath ${ }^{1}$ \\ ${ }^{1}$ Rwanda Military Hospital, Rwanda
}

\section{Background}

There is a lack of trained staff to respond to critically ill patients and cardiac and respiratory arrests in a health facility in Rwanda. This lack of human resources can lead to poor patient outcomes. Therefore, innovative strategies to provide the best and most appropriate patient care, with limited resources, is needed.

\section{Methods}

A group of nurses addressed the shortage of trained staff to respond to critical patients at Rwanda Military Hospital $(\mathrm{RMH})$ by developing a training program and a resuscitation response team. Members of the group who received the training when it was first initiated are now trainers, using the train the trainer model.

\section{Results}

All components of the training program are organized and delivered by RMH staff, the clinical mentor provided adjunct support only. Staff who trained together practiced with greater collaboration during actual resuscitation events and were more likely to initiate BLS if peer support was present. An increase in the number of admissions and transfers to the Intensive Care Unit were noted due to successful resuscitations.

\section{Conclusions}

Access to Advanced Cardiac Life Support training is necessary now that the cadre of Basic Life Support trained staff is growing. Increased access to training, peer support, and collaborative practice are effective strategies to strengthening resuscitation capacity within a hospital.

Key words: resuscitation, basic life support, capacity building, resuscitation, response teams, nurse trainer of trainers, critical care, Rwanda 